\title{
Instructions influence UR magnitude and conditioned alpha blocking
}

\author{
JOHN T. BRAGGIO \\ University of North Carolina, Asheville, North Carolina 28804 \\ and \\ R. THOMPSON PUTNEY \\ Georgia State University, Atlanta, Georgia 30303
}

\begin{abstract}
This study tried to assess the effects of instructions on conditioned alpha blocking. To do this, an equal number of college students were first assigned to an informed group (IG) or an uninformed group (UIG) and administered the same number and sequence of pseudoconditioning, conditioning, and extinction trials, using one of three different light intensities. The reinforcing property of a light US was also assessed by measuring UR magnitude in pseudoconditioning for individual subjects; the college students were later grouped with respect to the presence of low or high URs. It was found that UR magnitude, not US intensity, was predictive of conditioned alpha blocking. Nonetheless, conditioned alpha blocking occurred only for the subjects in the UIG who had low URs. For this group, conditioned alpha blocking was manifested as an increased effectiveness of the CS in blocking the alpha rhythm following a series of paired CS-US trials, and longer latencies of the alpha CR in conditioning and extinction as compared with the OR-produced responses in pseudoconditioning. The use of UR magnitude as a measure of the effectiveness of the US in producing conditioned alpha blocking and the effects of instructions on the conditionability of the EEG response system are discussed.
\end{abstract}

Following an early report by Cook and Harris (1937) showing that instructions can be as effective as paired CS-US trials in producing a GSR, there has been a revived interest in examining the role of instructions in classical conditioning (Dawson \& Furedy, 1976). The effects of instructions on CRs have been examined for different response systems, such as the GSR (Baer \& Fuhrer, 1968; Dawson \& Biferno, (1973), eyelid responses (Gormezano \& Moore, 1962), and HR/vasomotor responses (Shean, 1968). While it has been shown that the EEG response system can be classically conditioned (Jasper \& Shagass, 1941; Putney, 1973; Putney, Erwin, \& Smith, 1972), the effects of instructions on conditioned alpha blocking have not been examined.

Thus far, it has been shown that instructions can either facilitate (Dawson \& Furedy, 1976; Harvey \& Wickens, 1971; Hill, 1967) or inhibit the occurrence of CRs (Kimble, 1971; Norris \& Grant, 1948). It also has been reported that the effects of instructions on conditioned responses or CRs may be a function of US intensity (Gormezano \& Moore, 1962; Wickens, Allen, \& Hill, 1963; Wieland, Stein, \& Hamilton, 1963). Furthermore, Harvey and Wickens (1971)

A version of this paper was presented at the annual meeting of the Southeastern Psychological Association, Hollywood, Florida, May 7, 1977. Reprint requests should be sent to Dr. John T. Braggio, Department of Psychology, University of North Carolina at Asheville, University Heights, Asheville, North Carolina 28804. found that for the GSR response system, instructions influenced the magnitude of the UR, and it was through this link that CR efficacy may have been altered. Maltzman, Gould, Pendery, and Wolff (1977) reported that UR magnitude was an accurate predictor of CRs for a semantic conditioning study using the GSR response system. These investigators found better semantic conditioning in subjects showing effective UR magnitudes that were measured before the start of the conditioning trials. In a recently completed study, Braggio and Putney (1980) reported that for the EEG response system subjects with low UR magnitudes in pseudoconditioning demonstrated conditioned alpha blocking, while those with high UR magnitudes did not. These results suggest that the reinforcing effects of a given US may depend on one or more subject-defined factors of physiological activation that can be tapped by the UR magnitude factor prior to the start of the conditioning trials (Braggio \& Putney, 1980). The purpose of the present experiment was to determine if the conditionability of the EEG response system was influenced by instructions and light intensity; and, if so, were the effect(s) mediated by the UR magnitude variable?

\section{METHOD}

\footnotetext{
Subjects

From a presample of 34 college students, a total of 24 undergraduates with a median age of 22.4 years (range 18-28) were
} 
chosen to participate in this study. All of the subjects selected demonstrated, in their EEGs, persistent alpha spindles that lasted about $2.0 \mathrm{sec}$ and had a peak-to-peak amplitude of at least $35 \mu \mathrm{V}$.

\section{Apparatus}

The EEG was recorded on a Grass Model 78 electroencephalograph from the scalp of the subject, using gold electrodes placed over the left and right occipital areas $\left(\mathrm{O}_{1}, \mathrm{O}_{2}\right)$ and a reference electrode fastened to the right earlobe. The raw EEG, taken from the monopolar occipital lead with the most alpha, was filtered through a Krohn-Hite variable bandpass filter with the 3-dB points set at 7.5-12.5 Hz. After the filtered alpha was rectified (Grass 7P3 driver amplifier) and then integrated (Grass 7P10 polygraph integrator), it served as the input to the IBM-1800 data acquisition and control system computer. The filtered integrated alpha was recorded by the computer in millivolts (from the original microvolt values) every $.10 \mathrm{sec}$ during stimulus duration, which was always $3.0 \mathrm{sec}$. On each trial, 33 successive .10 -sec voltage values were recorded by the computer so that three .10-sec readings were taken before stimulus onset and 30 readings were taken during the 3.0-sec of stimulus duration.

For all subjects, the CS was a tone with a frequency of $450 \mathrm{~Hz}$ and an intensity of about $40 \mathrm{~dB}$ above the level of audibility. The intensities of the three USs were .602, 1.342, and $2.065 \log \mathrm{fL}$. The duration of the CSs and USs was always $3.0 \mathrm{sec}$. During conditioning, the interstimulus interval (ISI) was always $1.1 \mathrm{sec}$ and the intertrial interval (ITI) varied randomly from one trial to the next, but was never less than $10.0 \mathrm{sec}$.

The stimuli were presented while the subject sat in a comfortable reclining chair inside an industrial acoustics sound-attenuated chamber. The light stimuli were projected through the opaque window of the test chamber onto a circular target that had a diameter of $30 \mathrm{~cm}$. The tone was presented through a speaker that was centered immediately below the circular target. At stimulus offset, the subject was asked to depress an effortless response key. Voice contact was made possible from inside the soundattenuated chamber by the use of a two-way intercommunication system.

\section{Procedure}

The 24 subjects selected were assigned randomly to either the instructed group (IG) or the uninstructed group (UIG) based on their order of arrival to the laboratory. The subjects in the UIG were told only that the purpose of the experiment was to record their physiological reactions to different stimuli. However, the subjects in the IG were given an information sheet that summarized the purpose of the experiment. They were asked to read the instructions while the experimenter verbally described the purpose of the experiment. In brief, they were told that the tone and light stimuli would be presented during the test session. At first, the tone and light stimuli would not occur in any particular order. Then a tone would always precede a light stimulus for a predetermined number of trials. Finally, the tones and lights would be presented in a random order, again. Also, all subjects were asked to sit quietly in the sound-attenuated chamber and to avoid physical movement of arms, legs, and head, whenever possible.

Regardless of light US used, during the test session all subjects were given 102 trials. In pseudoconditioning, there were 36 trials that included a random presentation of 18 tone CSs and 18 light USs. The US trials consisted of six each of three different light intensities and were also randomized. During conditioning, $30 \mathrm{CS}-$ US trials were presented to the three groups, but, as was stated earlier, each group received just one light intensity as the US. In extinction, 36 trials of 18 CSs and 18 USs were again presented. As was the case in pseudoconditioning, the 18 US trials included six each of the three different light stimuli.

\section{RESULTS}

\section{UR Magnitude}

There are two ways to evaluate the reinforcing property of a light US on conditioned alpha blocking: The first is to define the reinforcing property of a light US in terms of an experimenter-defined factor (Braggio \& Putney, 1975). This manipulation is illustrated by the selection of light intensities that vary in brightness, as the use of low, medium, and high lights in the present study. The second way is to define the reinforcing property of a light US in terms of a subject-defined variable that reflects the differences in physiological activation (and corresponding functional thresholds) for a given stimulus parameter (Braggio \& Putney, 1980). In this study, both procedures were used to evaluate the reinforcing property of a light US in producing conditioned alpha blocking.

For all subjects, UR magnitudes were evaluated prior to the start of the conditioning trials, in pseudoconditioning, by examining the mean absolute voltage readings and the mean proportion reduction values for .70 through $1.00 \mathrm{sec}$ after light onset. The mean proportion reduction values were obtained by first computing the average of these four .10-sec voltage readings and then dividing this value by the session average of the three readings taken just before light onset.

Once a UR magnitude value was computed for the US-produced alpha block in pseudoconditioning, the subjects in each of the three US-intensity groups were ranked in ascending order with reference to UR magnitude and the median of each distribution was computed. The subjects who were below the median were assigned to the low UR (LUR) magnitude group, while those above the median were placed in the high UR (HUR) magnitude group. Once this grouping procedure was carried out, the light-produced alpha block in pseudoconditioning was analyzed in terms of the absolute voltage values and proportion reduction scores. It was found that light intensity was not a significant factor in blocking the alpha rhythm in pseudoconditioning for either the voltage measure $[\mathrm{F}(2,12)<1, \mathrm{p}\rangle .05]$ or the proportion reduction measure $[F(2,12)=2.154, p>.05]$. Therefore, from this point on, the experimenter-defined factor of light intensity was excluded from subsequent ANOVA results to be reported below.

The ANOVA design used to analyze the UR magnitude data consisted of Instructions (IG, UIG) by UR Magnitude (LUR, HUR) by Time of Stimulus Duration (.10 through $1.50 \mathrm{sec}$ after light onset) (Winer, 1962). 

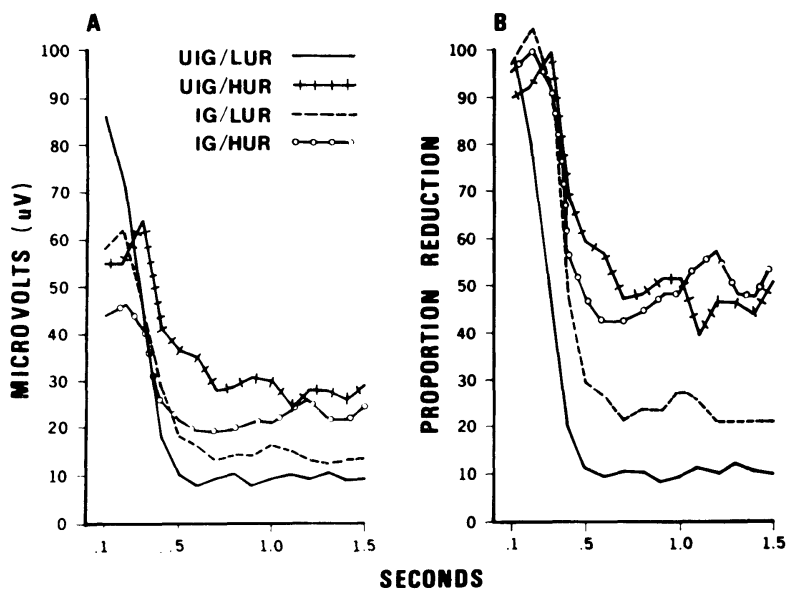

Figure 1. The light-produced UR block in pseudoconditioning is shown in terms of microvolts (A) and proportion reduction (B) for 15 successive .10-sec intervals following stimulus onset.

Analysis of the absolute voltage values for the lightproduced UR during the pseudoconditioning trials supported what can be seen in Figure 1A. It was found that the factors for UR Magnitude $[F(1,20)$ $=10.062, \mathrm{p}<.01]$, Instructions by UR Magnitude $[F(1,20)=4.772, p<.05]$, Stimulus Duration $[F(14,280)=87.011, p<.001]$, Instructions by Stimulus Duration $[\mathrm{F}(14,28)=2.757, \mathrm{p}<.001]$, UR Magnitude by Stimulus Duration $[F(14,280)=12.284$, $\mathrm{p}<.001$ ], and Instructions by UR Magnitude by Stimulus Duration $[F(14,280)=2.853, p<.001]$ were all significant, thereby suggesting that the two between-groups factors of Instructions and UR Magnitude work in opposite directions in determining the microvolt values for the base of the inverted " $\mathrm{J}$ " function for the light-produced alpha block. Group comparisons carried out by using the Newman-Keuls test (Winer, 1962) for values recorded for .70 through $1.00 \mathrm{sec}$ after light onset indicated that for the UIG, the subjects with LURs had less alpha (mean $=8.88$ ) than those subjects with HURs $($ mean $=29.42)$ $(p<.01)$. Also, the IG with LURs (mean $=14.33$ ) had significantly lower alpha than the IG with HURs $($ mean $=20.13)(p<.01)$. However, there were no significant differences between the UIG with LURs and the IG with LURs ( $p>.05)$. Also, there was not a significant correlation between the mean of the three prestimulus values recorded before light onset (not shown in Figure 1A) and the average voltage values for .70 through $1.00 \mathrm{sec}$ in the UR block $\left[r_{x y}(22)=.198, p>.05\right]$. There were no significant differences between the subjects with LURs and HURs $(p>.05)$. Therefore, these results suggest that, while there were differences in absolute voltage values taken before US onset in pseudoconditioning, these differences among the treatment means compared did not seem to affect the resting level of those values found at the base of the inverted " $J$ " curve.
To eliminate prestimulus baseline differences in absolute voltage values, these data were also analyzed using a proportion reduction measure, as described earlier. With this analysis, it is possible to evaluate the relative drop in alpha level with respect to each subject's prestimulus baseline value. Even though the absolute voltage values were transformed to relative scores, an inverted " $\mathrm{J}$ " function still described the light-produced alpha block for the pseudoconditioning trials. Statistical analyses of these data using the same ANOVA design described above showed that UR Magnitude $[\mathrm{F}(1,20)=26.479, \mathrm{p}<.001]$ was significant, indicating that the lights were more effective in blocking the alpha rhythm for the subjects with LURs than for those with HURs. Since the within-groups factor of Stimulus Duration $[F(14,280)$ $=80.252, \mathrm{p}<.001]$ and the interaction terms for UR Magnitude by Stimulus Duration $[F(14,280)=4.771$, $\mathrm{p}<.001]$ and Instructions by UR Magnitude by Stimulus Duration $[F(14,280)=2.139, p<.01]$ were significant, it can be concluded that the influence of the two between-groups factors of Instruction and UR Magnitude on the basal level of the inverted " $J$ " function differs as a function of the time in seconds after stimulus onset. However, it appears that the base of this inverted " $\mathrm{J}$ " function has leveled off by $.70 \mathrm{sec}$ after light onset. Therefore, differences in the light-produced block seem to start by about $.40 \mathrm{sec}$ after US onset and to level off by .70-1.00 sec.

The pattern of group differences found for the UR (when measured as a proportion reduction value) was the same as that described earlier for the absolute voltage units. Here, too, the UR was measured from .70 through $1.00 \mathrm{sec}$ after light onset. It was found that the mean proportion reduction for the subjects in the UIG with LURs (mean=9.41) was significantly lower than the mean for the subjects in the IG with LURs (mean $=23.54)(p<.01$; Newman-Keuls test, Winer, 1962), thereby suggesting that instructions decreased the effectiveness of a light US in blocking the alpha rhythm. No significant differences were found between the UIG with HURs and the IG with LURs ( $p>.05)$.

\section{Proportion Reduction}

The differential effects of Instructions and UR Magnitude on conditioned alpha blocking were examined for pseudoconditioning, conditioning, and extinction and analyzed by using the dependent measure of proportion reduction recorded for $1.10 \mathrm{sec}$ through $1.40 \mathrm{sec}$ after CS onset. Although the mean proportion reduction for 1.10-1.40 after CS onset was computed by using the three .10-sec intervals following US onset rather than the ISI, it is important to note that the UR was not included in this measurement since it occurred later in the interval. A careful inspection of the data reported by Braggio and Putney 


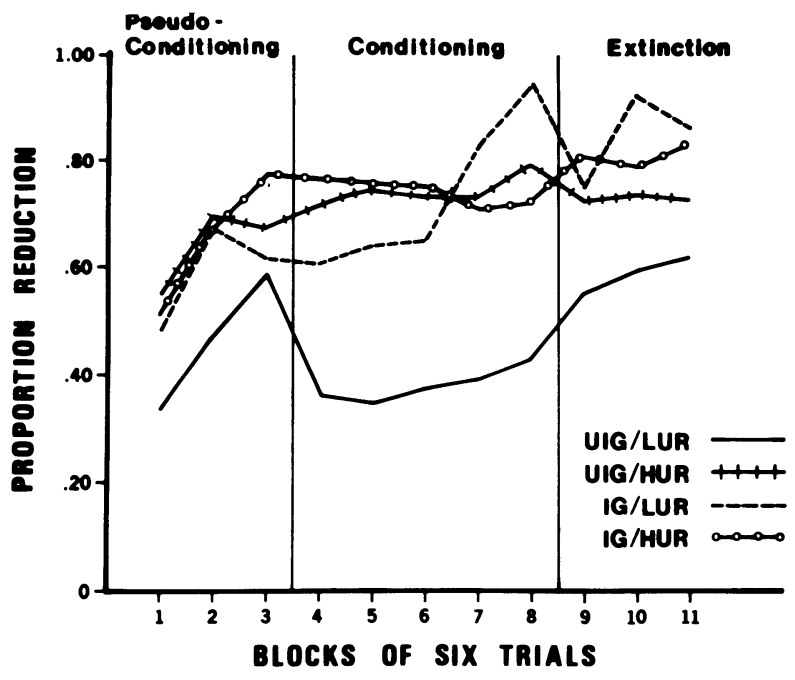

Figure 2. Proportion reduction values for the uninformed and informed subjects with either low or high UR magnitudes in pseudoconditioning (1-3 blocks of trials), conditioning (4-8 blocks of trials), and extinction (9-11 blocks of trials).

(1975) showed that the onset of the CR was separated in time from the onset of the UR by at least $.20 \mathrm{sec}$. In fact, the UR did not appear in the CS-US interval until $1.70 \mathrm{sec}$ after stimulus onset and .70 after light onset in pseudoconditioning.

Before statistical analyses were carried out, the individual trials for each subject were averaged into successive blocks of six trials. Since there was not a significant effect for the US Intensity factor $[F(2,12)$ $=2.790, \mathrm{p}>.05$ ], these data were analyzed using an ANOVA design consisting of Instruction (IG, UIG) by UR Magnitude (LUR, HUR) by Blocks of Trials (1-3 in pseudoconditioning, 4-8 in conditioning, and 9-11 in extinction). These data are shown in Figure 2. As seen here, it was found that the Instruction $[F(1,10)$ $=7.963, p<.05]$, UR Magnitude $[F(1,10)=6.408$, $\mathrm{p}<.05]$, Blocks of Trials $[\mathrm{F}(10,200)=6.538, \mathrm{p}<.01]$, and the corresponding interaction of Instructions by UR Magnitude $[F(1,20)=5.410, p<.05]$ were significant, thereby suggesting that the effects of these two between-groups factors of Instructions and UR Magnitude did have an obvious effect on the conditioned alpha blocking curve. In pseudoconditioning, all groups showed habituation to the CS-only trials, since the proportion reduction for the third block $($ mean $=.66)$ was significantly higher than that for the first block (mean $=.47)(\mathrm{p}<.01)$. Also, by the third block of pseudoconditioning trials, the UIG with LURs was not significantly different from the IG with LURs ( $p>.05)$; at the same time, there was not a significant difference between the IG with LURs and the UIG with HURs ( $p>.05)$.

In conditioning, only the uninformed subjects with LURs showed evidence of conditioned alpha blocking. For the $\mathbf{3 0}$ conditioning trials, this group had a significantly lower proportion reduction value than either one of the means for the three remaining groups (in each case, $p<.01$ ). Furthermore, the form of the conditioning curves differed for the two informed and uninformed groups with LURs: The conditioning curve for the UIG with LURs took the shape of a decreasing monotonic function across the first two blocks of CS-US trials ( $p<.05$ ), which was then followed by a plateau that was significantly lower than the mean proportion reduction value observed for the last block of pseudoconditioning trials $(p<.01)$. However, for the IG with LURs, there was an increasing monotonic function $(p<.01)$ that resembled an extension of the habituation curve observed in pseudoconditioning. Finally, the UIG with LURs had a significantly lower proportion reduction value for the first block of extinction trials than the other three groups $(p<.01$, in all cases) shown in Figure 2. Therefore, these results clearly showed that conditioned alpha blocking occurred for those college students who were uninformed and had LURs.

\section{Latency of CS-Produced Alpha Block}

To carry out this analysis, the latency of the smallest value occurring from .20 to $1.20 \mathrm{sec}$ after tone onset was recorded for each trial. Then, for each subject, the individual trials were averaged in blocks of six. At that point, statistical analyses were carried out. Since there was not a significant effect for US intensity $[F(2,12)=3.028, p>.05]$, the latency scores were analyzed using an ANOVA design consisting of Instructions (UIG, IG) by UR Magnitude (LUR, HUR) by Blocks of Trials (1-3 in pseudoconditioning, 4-8 in conditioning, and 9-11 in extinction), as shown in Figure 3. It was found that the Instruction $[F(1,20)$ $=6.450, p<.05]$ and UR Magnitude $[F(1,20)=16.690$,

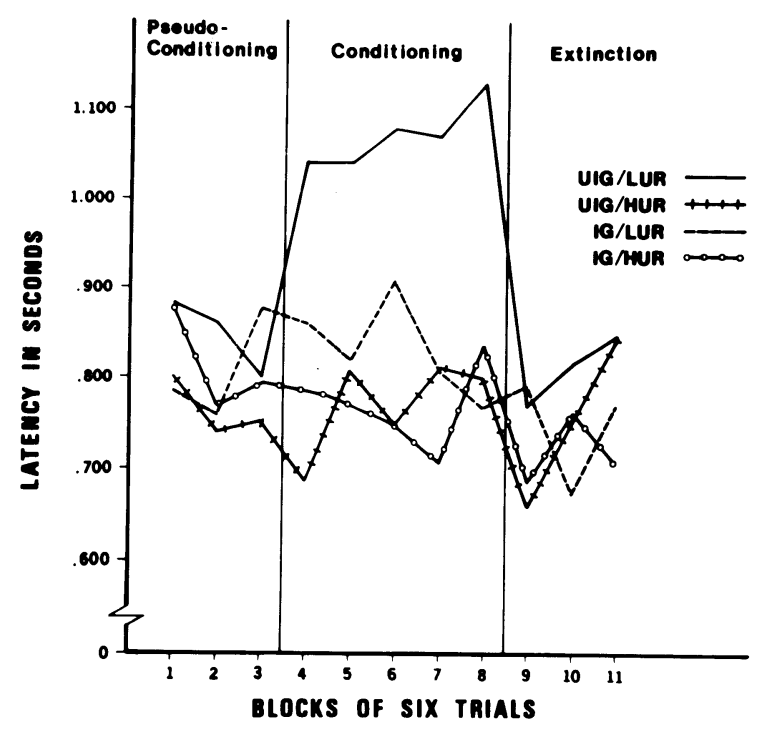

Figure 3. Latency of the CS-produced alpha block for the uninformed and informed subjects with either low or high UR magnitudes in pseudoconditioning (1-3 blocks of trials), conditioning (4-8 blocks of trials), and extinction (9-11 blocks of trials). 
$\mathrm{p}<.001]$ factors were significant, as was the corresponding interaction term for Instruction by UR Magnitude $[F(1,20)=7.771, p<.05]$. Latency scores changed as a function of the Trials factor $[\mathrm{F}(10,200)$ $=3.239, \mathrm{p}<.001 \mathrm{l}$, suggesting that conditioned alpha blocking was accompanied by longer latencies, while habituation was characterized by shorter latencies. The significant interaction of UR Magnitude by Blocks of Trials $[F(10,200)=2.203, p<.05]$ suggests that, like the EEG CRs, the latency function became progressively longer as more paired CS-US trials were administered.

Individual comparisons among the treatment means indicated that there was a significantly shorter mean latency value for the second (mean $=.78)$ and third $($ mean $=.80)$ as compared with the first $($ mean $=.84)$ block of pseudoconditioning trials (in both cases, $\mathrm{p}<.01$ ). For the last block of pseudoconditioning trials, there were no significant differences between the informed subjects with HURs and the uninformed subjects with LURs $(p>.05)$. The uninformed subjects with HURs showed a significantly shorter latency $($ mean $=.75)$ than the instructed subjects with LURs or the ones in the remaining two groups (in each case, $\mathrm{p}<.01$ ). During conditioning, it was found that only the uninstructed subjects with LURs had significantly longer latencies than the other three groups (in all cases, $\mathrm{p}<.01$ ). Latency scores for this group were significantly longer in conditioning than they were for the last block of pseudoconditioning trials $(\mathrm{p}<.05)$. Also, it was found that the informed subjects with LURs had latency scores that were significantly shorter in conditioning than in the last block of pseudoconditioning trials $(p<.01)$. In extinction, the mean latency scores for the two informed groups having either LURs or HURs, as well as the uninformed subjects with HURs, were significantly shorter for the first block of extinction trials than for the last block of pseudoconditioning trials (in all cases, $\mathrm{p}<.05$ ), thereby suggesting that the paired CS-US trials functioned only as a further continuation of the habituation curve observed earlier in pseudoconditioning. In contrast, the uninformed subjects with LURs did not show significantly lower latency scores in extinction than those found in pseudoconditioning; these results suggest that, for this group of subjects, the conditioning trials attenuated the continuation of the habituation curve. In conclusion, these data suggest that the shorter latencies for CSonly trials that were observed in pseudoconditioning may reflect ORs (i.e., a unitary nonspecific response that is elicited by a novel stimulus), while the longer ones seen in conditioning may be indicative of CRs (Sokolov, 1963).

\section{DISCUSSION}

The results of the present study have shown that instructions prevent the appearance of conditioned alpha blocking by decreasing the effectiveness of a light US in blocking the alpha rhythm. Analysis of the conditioning data indicated that a tone CS became more effective in blocking the alpha rhythm after it was paired with a light US only for the uninformed subjects that had LURs (see Figure 2). Unlike other conditioning studies (Gormezano \& Moore, 1962; Wickens et al., 1963; Wieland et al., 1963), in this experiment US intensity did not eliminate the effects of instructions on CR efficacy, since there was not a significant effect for this factor on conditioned alpha blocking when measured as proportion reduction values or latency scores (see Figures 2 and 3 ).

In this study and in an earlier report (Braggio \& Putney, 1975), it was found that lights suppressed the alpha rhythm more than did tones. However, for these data, the latency for the CS- and US-only trials in pseudoconditioning was identical (mean $=.810 \mathrm{sec}$ ) and not significantly different from each other. Thus, these latency scores for unpaired tone and light stimuli may reflect the occurrence of ORs in the pseudoconditioning portion of the experiment (Sokolov, 1963).

Nonetheless, it should be remembered that the light USs were still more effective in blocking the alpha rhythm than were the tone CSs. In conditioning, the CS increased in its effectiveness in blocking the alpha rhythm after it was paired with a US, and only for those subjects that were uninformed and had LURs. For these subjects, the lights used as USs functioned as effective stimuli that were able to produce conditioned alpha blocking. Furthermore, as more conditioning trials were administered, there was a transformation in the latency of the CS-produced alpha block so that it became longer during conditioning and extinction and shorter during pseudoconditioning. Therefore, these results suggest that the conditioning process produced changes in amplitude and latency characteristics of the EEG CRs by making the conditioned responses different from the tone- or light-only responses found in pseudoconditioning.

This CR amplitude and latency pattern occurred only for the uninformed subjects with LURs. Also, conditioned alpha blocking occurred for the uninformed subjects with low UR magnitudes and not for any one of the other three groups. In part, these results replicate the findings reported by Braggio and Putney (1980) for the EEG response system. Other investigators have also found that UR magnitude is an accurate predictor of CR efficacy (Razran, 1957). In a review of the Soviet classical conditioning literature, Razran (1957) reported that UR magnitude is used as a measure of US effectiveness. In this country, it has been found that UR magnitude is also predictive of CR efficacy (Maltzman et al., 1977). Therefore, it may be concluded that UR magnitude can be used as a way of assessing whether or not a light stimulus functions as an effective US in producing conditioned alpha blocking or generating CRs for other classically conditioned response systems. 
It is important to note that, in the present study, UR magnitude was not found to be a measure of generalized activation, as suggested by Morgenson and Martin (1968), since the initial alpha baseline value was not related to the measure of UR magnitude taken at the base of the inverted " $\mathrm{J}$ " function (see Figure 1). Also, greater amounts of alpha blocking were produced by the light (and not the tone) stimuli in pseudoconditioning. If the occurrence of conditioned alpha blocking were due to a generalized increase in physiological activity, then one would have expected that the individuals with LURs, regardless of instructions, should have demonstrated greater amounts of alpha blocking to the USs and CSs during the conditioning trials. Because the subjects with LURs showed response specificity to the light stimuli, these results suggest that UR magnitude may be a specific index of physiological activation, tapping one or more factors that are indicative of the reinforcing property of a US, or of the capacity of a stimulus in blocking the alpha rhythm.

Given the finding that UR magnitude may be used as a measure of the effectiveness of a light stimulus in producing conditioned alpha blocking, it is then possible to see how instructions influence CRs. These results suggest that instructions first influence UR magnitude by decreasing the reinforcing property of a light stimulus in blocking the alpha rhythm. In turn, less effective USs produce higher UR magnitudes that prevent the occurrence of conditioned alpha blocking. Therefore, it can be concluded that, for the EEG response system, the effects of instructions on CRs are mediated by UR magnitude.

A similar relationship between instructions, UR magnitude, and CR efficacy has been observed for the GSR response system. Harvey and Wickens (1971) reported that, for the GSR response system, instructions influenced CR efficacy through UR magnitude. Maltzman and his associates (Maltzman et al., 1977) also found that it was possible to use UR magnitude as a way to predict the occurrence of CRs in a semantic conditioning study of the GSR response system. These investigators found better semantic conditioning for the subjects who demonstrated effective UR magnitudes prior to the start of the conditioning study. Finally, UR magnitude, when measured as the OR to a US, was also predictive of CR efficacy (Fuhrer, Baer, \& Cowan, 1973).

In summary, the results of the present study suggest that the effectiveness of a US in producing conditioned alpha blocking can be influenced by both UR magnitude and instructions. Alpha desynchronization, when measured as a UR magnitude variable and/or a latency factor, may reflect a subject's unique physiological response to a US. By presenting one or more light stimuli of given intensities and observing the occurrence of systematic patterns of high or low levels of alpha desynchronization (i.e., HURs and LURs), it is then possible to better define the psychological and physiological significance of an externally defined stimulus for each human subject used in a classical conditioning study. Such information may be used to predict the rate at which a tone or light stimulus no longer blocks the alpha rhythm (e.g., habituation). In the final analysis, whether or not a given light US produces conditioned alpha blocking may depend on the interaction of the kind and/or intensity of the US, awareness of the conditioning process produced by instructions or prior knowledge, and the subject's specific physiological level of activation as defined by low or high UR magnitude.

\section{REFERENCES}

Baer, P. E., \& Fuhrer, M. J. Cognitive processes during differential trace and delayed conditioning of the GSR. Journal of Experimental Psychology, 1968, 78, 81-88.

Braggio, J. T., \& Putney, R. T. The effects of unconditioned stimulus intensity on conditioned alpha blocking. Physiological Psychology, 1975, 3, 355-362.

Braggio, J. T., \& Putney, R. T. UR magnitude as a predictor of conditioned alpha blocking. Psychophysiology, 1980, 17, 38-44.

Cook, S. W., \& Harris, R. E. The verbal conditioning of the galvanic skin reflex. Journal of Experimental Psychology, 1937, 22, 202-217.

Dawson, M. E., \& Biferno, M. A. Concurrent measurement of awareness and electrodermal conditioning. Journal of Experimental Psychology, 1973, 101, 55-62.

Dawson, M. E., \& Furedy, J. J. The role of awareness in human differential autonomic classical conditioning: The necessary-gate hypothesis. Psychophysiology, 1976, 13, 50-53.

Fuhrer, M. J., Baer, P. E., \& Cowan, C. O. Orienting responses and personality variables as predictors of differential conditioning of electrodermal responses and awareness of stimulus relations. Journal of Personality and Social Psychology, 1973, 27, 287-296.

Gormezano, I., \& Moore, J. W. Effects of instructional set and UCS intensity on the latency, percentage, and form of the eyelid response. Journal of Experimental Psychology, 1962, 63, 487-494.

Harvey, B., \& Wickens, D. D. Effects of instructions on responsiveness to the CS and UCS in GSR conditioning. Journal of Experimental Psychology, 1971, 87, 137-140.

HiLl, F. A. Effect of instructions and subject's need for approval on the conditioned galvanic skin response. Journal of Experimental Psychology, 1967, 73, 461-467.

JASPer, H., \& Shagass, C. Conditioning of the occipital alpha rhythm in man. Journal of Experimental Psychology, 1941, 28, 373-387.

Kimble, G. A. Cognitive inhibition in classical conditioning. In H. H. Kendler \& J. T. Spence (Eds.), Essays in neobehaviorism: A memorial to Kenneth W. Spence. New York: AppletonCentury-Crofts, 1971.

Maltzman, I., Gould, J., Pendery, M., \& Wolff, C. Semantic conditioning and generalization of the galvanic skin responseorienting-reflex with overt and covert activity. Journal of Experimental Psychology: General, 1977, 106, 172-184.

Morgenson, D. F., \& Martin, I. The orienting response as a predictor of autonomic conditioning. Journal of Experimental Research in Personality, 1968, 2, 89-98. 
Norris, E. B., \& Grant, D. A. Eyelid conditioning as affected by verbally induced inhibitory set and counter reinforcement. American Journal of Psychology, 1948, 61, 37-49.

Putney, R. T. Conditioned alpha blocking re-examined with the measurement of individual wave amplitudes. Electroencephalography and Clinical Neurophysiology, 1973, 34, 485-493.

Putney, R. T., Erwin, T. J., \& Smith, T. S. The facilitation of conditioned alpha blocking with an overt response. Psychonomic Science, 1972, 26, 16-18.

RAZRAN, G. The dominance-contiguity theory of the acquisition of classical conditioning. Psychological Bulletin, 1957, 54, 1-46.

Shean, G. C. Vasomotor conditioning and awareness. Psychophysiology, 1968, 5, 22-30.
Sokolov, E. N. Perception and the conditioned reflex. New York: Macmillan, 1963.

Wickens, D. D., Allen, C. K., \& Hill, F. A. Effects of instructions and UCS strength on extinction of the conditioned GSR. Journal of Experimental Psychology, 1963, 66, 235-240.

Wieland, W. F., Ste in, M., \& Hamilton, C. L. Intensity of the unconditioned stimulus as a factor in conditioning out awareness. Psychosomatic Medicine, 1963, 25, 124-132.

WineR, B. J. Statistical principles in experimental design. New York: McGraw-Hill, 1962.

(Received for publication January 31, 1979; revision accepted April 25, 1980.) 